\title{
BaRTv1.0: an improved barley reference transcript dataset to determine accurate changes in the barley transcriptome using RNA-seq
}

Paulo Rapazote-Flores ${ }^{1}$, Micha Bayer ${ }^{1}$, Linda Milne ${ }^{1}$, Claus-Dieter Mayer ${ }^{2}$, John Fuller ${ }^{3}$, Wenbin Guo ${ }^{4}$, Pete E. Hedley ${ }^{3}$, Jenny Morris ${ }^{3}$, Claire Halpin ${ }^{4}$, Jason Kam ${ }^{4,5}$, Sarah M. McKim ${ }^{4}$, Monika Zwirek ${ }^{4,6}$, M. Cristina Casao ${ }^{4}$, Abdellah Barakate ${ }^{3}$, Miriam Schreiber ${ }^{3}$, Gordon Stephen ${ }^{1}$, Runxuan Zhang ${ }^{1}$, John W. S. Brown ${ }^{3,4}$,

Robbie Waugh ${ }^{3,4}$ and Craig G. Simpson ${ }^{3^{*}}$ (D)

\begin{abstract}
Background: The time required to analyse RNA-seq data varies considerably, due to discrete steps for computational assembly, quantification of gene expression and splicing analysis. Recent fast non-alignment tools such as Kallisto and Salmon overcome these problems, but these tools require a high quality, comprehensive reference transcripts dataset (RTD), which are rarely available in plants.
\end{abstract}

Results: A high-quality, non-redundant barley gene RTD and database (Barley Reference Transcripts - BaRTv1.0) has been generated. BaRTV1.0, was constructed from a range of tissues, cultivars and abiotic treatments and transcripts assembled and aligned to the barley cv. Morex reference genome (Mascher et al. Nature; 544: 427-433, 2017). Fulllength cDNAs from the barley variety Haruna nijo (Matsumoto et al. Plant Physiol; 156: 20-28, 2011) determined transcript coverage, and high-resolution RT-PCR validated alternatively spliced (AS) transcripts of 86 genes in five different organs and tissue. These methods were used as benchmarks to select an optimal barley RTD. BaRTv1.0Quantification of Alternatively Spliced Isoforms (QUASI) was also made to overcome inaccurate quantification due to variation in $5^{\prime}$ and $3^{\prime}$ UTR ends of transcripts. BaRTV1.0-QUASI was used for accurate transcript quantification of RNA-seq data of five barley organs/tissues. This analysis identified 20,972 significant differentially expressed genes, 2791 differentially alternatively spliced genes and 2768 transcripts with differential transcript usage.

Conclusion: A high confidence barley reference transcript dataset consisting of 60,444 genes with 177,240 transcripts has been generated. Compared to current barley transcripts, BaRTv1.0 transcripts are generally longer, have less fragmentation and improved gene models that are well supported by splice junction reads. Precise transcript quantification using BaRTv1.0 allows routine analysis of gene expression and AS.

Keywords: Barley, Reference transcript dataset, Transcriptome, Differential gene expression, Differential alternative splicing

\footnotetext{
* Correspondence: craig.simpson@hutton.ac.uk

${ }^{3}$ Cell and Molecular Sciences, The James Hutton Institute, Invergowrie,

Dundee DD2 5DA, UK

Full list of author information is available at the end of the article
}

(c) The Author(s). 2019 Open Access This article is distributed under the terms of the Creative Commons Attribution 4.0 International License (http://creativecommons.org/licenses/by/4.0/), which permits unrestricted use, distribution, and reproduction in any medium, provided you give appropriate credit to the original author(s) and the source, provide a link to the Creative Commons license, and indicate if changes were made. The Creative Commons Public Domain Dedication waiver (http://creativecommons.org/publicdomain/zero/1.0/) applies to the data made available in this article, unless otherwise stated. 


\section{Background}

Barley is an important cereal crop grown across a geographical range that extends from the Arctic Circle to the hot and dry regions of North Africa, the near east and equatorial highlands. Adaptation of barley to very different growing conditions reflects important characteristics of genomic and transcriptomic diversity that leads to the success of the crop at different latitudes [3, 16 , 47]. Changes in gene expression during development and in response to daily and seasonal environmental challenges and stresses drive re-programming of the barley transcriptome [11, 26, 27, 30, 46]. Transcriptomes consist of complex populations of transcripts produced through the co-ordinated transcription and post-transcriptional processing of precursor messenger RNAs (pre-mRNAs). Alternative splicing (AS) of pre-mRNA transcripts is the main source of different transcript isoforms that are generated through regulated differential selection of alternative splice sites on the pre-mRNA and up to $60-70 \%$ introncontaining plant genes undergo AS [11-14, 19, 31, 34, 36, $54,55]$. The two main functions of AS are to increase protein diversity and regulate expression levels of specific transcripts by producing AS isoforms that are degraded by nonsense mediated decay (NMD) [28, 32, 39, 45, 54]. Extensive AS has been reported in barley [11, 26, 41, $59,60]$ and allelic diversity further contributes to the landscape of AS transcript variation among genotypes through elimination and formation of splice sites and splicing signals [22, 33, 50].

Although RNA-seq is the current method of choice to analyse gene expression, major problems exist in the computational assembly and quantification of transcript abundance from short read data with widely used programs. Such assemblies are typically inaccurate because first, they generate a large proportion of mis-assembled transcripts and second, they fail to assemble thousands of real transcripts present in the sample dataset [25, 43]. In contrast, non-alignment tools such as Kallisto and Salmon $[6,42]$ provide rapid and accurate quantification of transcript/gene expression from RNA-seq data. However, they require high quality, comprehensive transcript references, which are rarely available in plants [7]. In barley, RNA-seq data from eight different barley organs and tissues from the variety Morex, a six-rowed North American cultivar, was used to support annotation of the first barley genome sequence [26]. The subsequent release of the barley pseudogenome, estimated to contain $98 \%$ of the predicted barley genome content, has 42,000 highconfidence and 40,000 low-confidence genes and ca. 344, 000 transcripts [35]. However, detailed analysis of individual gene models in the pseudogenome shows that the current annotation contains a high frequency of chimeric and fragmented transcripts that are likely to negatively impact downstream genome-wide analyses of differential expression and AS. In Arabidopsis, a diverse, comprehensive and accurate Reference Transcript Dataset (AtRTD2), was constructed from short read RNA-seq data by assembling transcripts with the assembly functions of Cufflinks and Stringtie, followed by multiple stringent quality control filters. These filters removed poorly assembled transcripts (e.g. with unsupported splice junctions), transcript fragments and redundant transcripts, all of which affected the accuracy of transcript quantification by Salmon/Kallisto [61, 62]. AtRTD2 has been used for genome-wide differential expression/differential AS to identify novel regulators of the cold response and splicing factors that regulate AS in innate immunity and root development [4, $9,10,63]$.

Here, we describe the development of a first barley reference transcript dataset and database (Barley Reference Transcripts - BaRTv1.0) consisting of 60,444 genes and 177,240 non-redundant transcripts. To create BaRTv1.0, we used 11 different RNA-seq experimental datasets representing 808 samples and 19.3 billion reads that were derived from a range of tissues, cultivars and treatments. We used high-resolution RT-PCR (HR RT-PCR) results to optimise parameters for transcript assembly and to validate differential AS in five different barley organs and tissues. We further compared the BaRTv1.0 transcripts to 22,651 Haruna nijo full-length (fl) cDNAs [37] to assess the completeness and representation of the reference transcript dataset. As in Arabidopsis, we also generated a version of the RTD specifically for quantification of alternatively spliced isoforms (BaRTv1.0-QUASI) for accurate expression and AS analysis, which overcomes inaccurate quantification due to variation in the $5^{\prime}$ and $3^{\prime} \operatorname{UTR}[53,61]$. Finally, we used BaRTv1.0-QUASI to explore RNA-seq data derived from five diverse barley organs/tissues identifying 20,972 differentially expressed genes and 2791 differentially alternatively spliced genes amongst the samples.

\section{Results}

Transcript assembly and splice site determination

To maximise transcript diversity in the barley RTD assembly we selected barley Illumina short read datasets that covered different barley varieties, a range of organs and tissues at different developmental stages and plants/ seedlings grown under different abiotic stresses. The datasets represent 11 different RNA-seq experiments, containing 808 samples and 19.3 billion reads (Additional file 1: Table S1). Most samples consisted of paired-end reads that were between 90 and $150 \mathrm{bp}$ in length and produced from Illumina HiSeq 2000 or Illumina HiSeq 2500 runs. Exceptions were the dataset from Golden Promise anthers and meiocytes, which contained over 2 billion paired end $35-76$ bp reads and the internode dataset which contained unpaired $100 \mathrm{bp}$ reads (Additional file 1: Table S1). The raw RNA-seq data of all samples were quality controlled, 
trimmed and adapters removed using FastQC and Trimmomatic (Fig. 1; Additional file 1: Table S1). Reads were mapped to the reference genome sequence of barley cv. 'Morex' (Hv_IBSC_PGSB_v2) [35] using STAR (Spliced Transcripts Alignment to a Reference) software [17, 18] (Fig. 1). To improve mapping accuracy and filter out poorly supported splice junctions from the sequence reads, while also considering the variability of expression levels in the different samples, we performed a three-pass STAR mapping (Additional file 2: Figure S1). This was based on a two-pass alignment method to increase splice junction alignment rate and sensitivity by performing a high-stringency first pass with STAR, which was then used as annotation for a second STAR pass at a lower stringency alignment [57]. We also performed a less stringent third pass with STAR to capture further splice junction read number evidence from the range of barley datasets that included different cultivars and landraces, which will show sequence variation among reads and affect their mapping. The third pass did not allow any additional splice junctions to be generated that were not already present after the second pass. The advantage of the third pass was to allow more reads to map to the splice junction and increase support for rarer splice site selections and increase transcript diversity. (See Materials and Methods). The number of uniquely mapped reads after the three
STAR passes ranged from 73 to $85 \%$ (data not shown) across the 11 experiments. This iterative alignment and filtering process using STAR produced a robust splice junction reference dataset of 224,654 splice junctions that was used to support the identification of multiple transcripts per gene.

\section{Optimisation of cv. Morex guided reference transcript assemblies}

Transcriptomes for each of the 808 samples were assembled using StringTie [43] and different parameter combinations tested to optimise the quality and number of transcripts (Fig. 2; Additional file 1: Table S2). Throughout this process the quality of the Morex referencebased transcript assemblies were benchmarked against data from a HR RT-PCR panel of 86 primer sets covering 83 genes and 220 transcripts [51] to accurately analyse the proportion of alternatively spliced products in a subset of the cv. Morex experimental samples (Developing inflorescences INF1 and INF2, leaf shoots from seedlings - LEA, embryo - EMB, internode - NOD - see Materials and Methods). The primer list is available at https://ics.hutton. ac.uk/barleyrtd/primer_list.html (Additional file 1: Table S3). At each stage the spliced proportions from HR RTPCR were compared to the spliced proportions of the same AS event(s) derived from the Transcripts Per Million

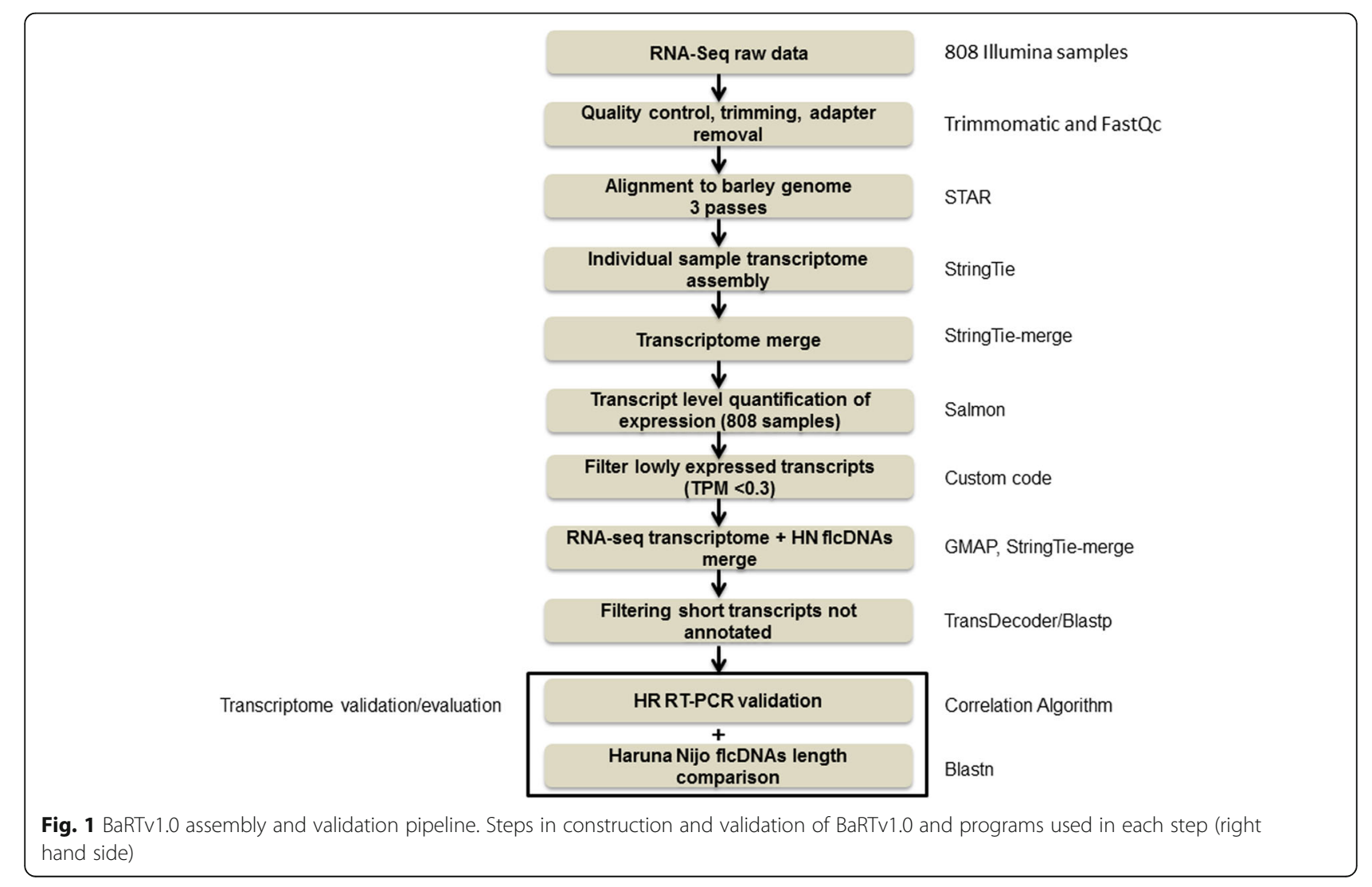



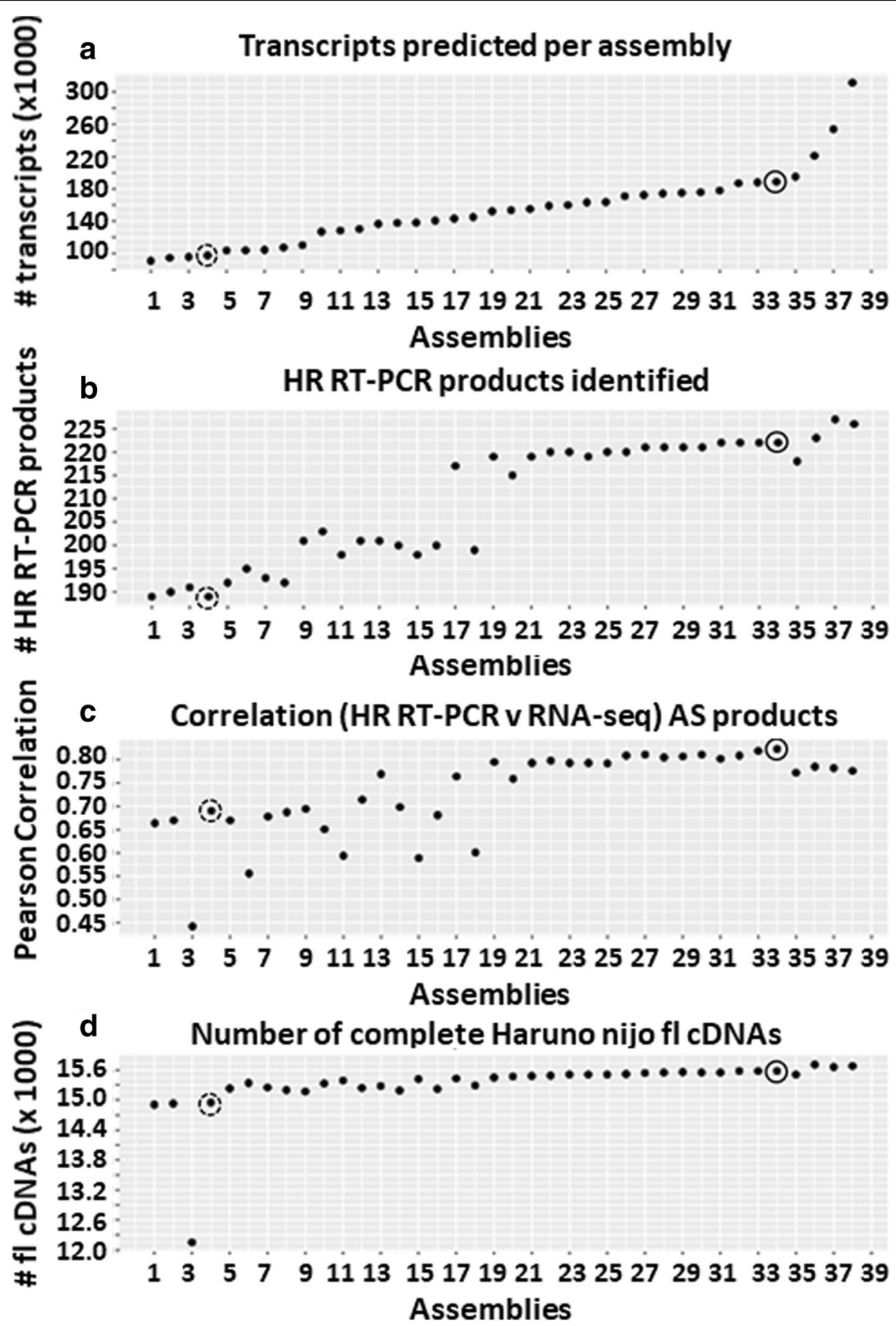

Fig. 2 Benchmarking of 38 different StringTie Morex reference-based assemblies. The four plots show different benchmark tests to assess the parameters used in the StringTie assemblies. The graphs do not start from 0 on the y axis. a Transcript number; $\mathbf{b}$ the number of HR RT-PCR products that match transcripts; c correlation of the proportions of transcripts in 86 AS events derived from HR RT-PCR and the RNA-seq data using the different assemblies as reference for transcript quantification by Salmon; and $\mathbf{d}$ the number of Haruna nijo fl cDNAs that match RTD transcripts. Each plot point represents the result of a StringTie assembly using different parameters (Additional file 1: Table S2). The broken circled plot points at assembly 4, an assembly using STAR defaults (without splice junction filtering) and StringTie defaults. The solid circled plot point at assembly 34 represents the selected optimised StringTie parameters used to produce BaRTv1.0 (see also Materials and Methods; Additional file 2: Figure S3; Additional file 1: Table S2) 
(TPM) counts extracted from the RNA-seq data analysis $[51,61]$ using an automated method (see Fig. 1; Materials and Methods for description and https://github.com/PauloFlores/RNA-Seq-validation for script).

Each StringTie assembly was further compared to the 22,651 Haruna nijo full-length fl cDNAs [37] to assess both the completeness and representation. Of these, 17, 619 (81.2\%) fl cDNAs had at least 90\% coverage and 90\% sequence identity with transcripts in the RTD using BLASTn [2] (Additional file 2: Figure S2). These fl cDNAs were used to quantify coverage in the optimisation of assemblies with StringTie (Fig. 2; Additional file 1: Table S2).

Among the different StringTie parameters tested, the read coverage ("-c" parameter) was found to be important and a value of 2.5 was selected as the optimum. A lower read coverage value induced fragmentation, greatly increasing the number of genes, fewer matching RTPCR products, poorer correlation with the HR RT-PCR data and reduced matching to the Haruna nijo fl cDNAs (Fig. 2, for example assemblies 9-16; Additional file 1: Table S2), while a value of 3 led to a lower number of genes and transcripts being defined (Fig. 2, for example assemblies 26-30; Additional file 1: Table S2). The isoform-fraction ("-f" parameter) was optimal at 0 , maximising the number of transcripts, while still maintaining a strong correlation with HR RT-PCR data and high numbers of matching Haruna nijo fl cDNAs (Fig. 2, assemblies 17, 19-38; Additional file 1: Table S2). A minimum locus gap separation value (“-g” parameter) of 50 bp was selected as an optimum value. Values greater than $50 \mathrm{bp}$ led to the prediction of fewer transcripts and poorer correlation with the HR RT-PCR data, although there was a small improvement in the coverage of the Haruna nijo $\mathrm{fl} \mathrm{cDNAs}$. Increasing the gap separation to $500 \mathrm{bp}$ forced distinct genes to merge resulting in longer transcripts, poorer similarity with Haruna nijo fl cDNAs and very poor correlation with the HR RT-PCR data due to the creation of chimeric genes (Fig. 2; in assembly 3). The improvement in the assemblies with the optimised StringTie parameters is illustrated by comparison to the assembly produced using StringTie default parameters (Fig. 2). The optimised assembly had a $14 \%$ increase in splice product detection in the HR RT-PCR analysis (220 versus 189 RT-PCR products) and increased Pearson correlation values from 0.60 to 0.79 between the RNA-seq data and HR RT-PCR data. It also recovered 634 more complete Haruna nijo fl cDNAs compared to the StringTie assembly run in default mode.

\section{Construction of BaRTv1.0}

Having established optimal assembly parameters, to construct the RTD, transcripts were merged to create a single set of non-redundant transcripts. The dataset was filtered to remove poorly expressed transcripts $(<0.3$ TPM) and then merged with the genome-mapped Haruna nijo full-length cDNAs (Fig. 1). Finally, we used TransDecoder [24] to predict protein coding regions and BLASTp [2] to filter out transcripts equal to or less than 300 bps long (8767 transcripts) and showing less than $70 \%$ protein coverage and identity with the Poaceae reference protein dataset (Fig. 1), which removed all but 25 transcripts of less than $300 \mathrm{bp}$ (Additional file 2: Figure S4). After merging and filtering, we retained 224,654 unique splice junctions, 60,444 genes and 177,240 transcripts to establish the non-redundant reference transcript dataset named BaRTv1.0 (Table 1).

Previous studies in Arabidopsis and human RNA-seq analysis showed that variation in the $5^{\prime}$ and $3^{\prime}$ ends of assembled transcript isoforms of the same gene affected accuracy of transcript quantification. Variation may be the result of legitimate transcriptional and post-transcriptional activity. However, transcript variation may also result from aberrant reverse transcription/internal priming during library preparation, RNA degradation and mis-assembly or mis-annotation during transcript assembly [53, 61]. This was overcome by padding shorter $5^{\prime}$ and $3^{\prime}$ ends to the $5^{\prime}$ and $3^{\prime}$ ends of the longest gene transcript [61, 63]. We similarly modified BaRTv1.0 to produce transcripts of each gene with the same $5^{\prime}$ and $3^{\prime}$ ends to generate BaRTv1.0QUASI specifically for transcript and AS quantification. Both datasets are available for download from https://ics. hutton.ac.uk/barleyrtd/downloads.html. An additional copy of the RTD is available in the Zenodo repository (https:// doi.org/10.5281/zenodo.3360434). In addition, a website was created to visualise individual BaRT transcripts, access transcript sequences, and allow for BLAST searching and comparison with existing HORVU transcripts [35] https:// ics.hutton.ac.uk/barleyrtd/index.html.

\section{BaRTv1.0 represents an improved barley transcript dataset}

The barley cv. Morex pseudo-molecule sequences were accompanied by a set of ca. $344 \mathrm{k}$ HORVU transcripts [35], nearly double the number in BaRTv1.0. Close inspection of the HORVU transcripts identified short, fragmented and redundant transcripts. The quality control filters used in the construction of BaRTv1.0 aimed to reduce the number of transcript fragments and redundancy as these negatively impact the accuracy of transcript quantification [61]. The BaRTv1.0 and HORVU datasets were directly compared with the numbers of complete Haruna nijo fl cDNAs and correlating the proportions of AS transcript variants measured by HR RT-PCR with those derived from the RNA-seq analysis (Additional file 1: Table S4). The BaRTv1.0 transcript dataset identified more of the experimentally determined HR RT-PCR products (220 versus 191) and has higher Pearson and Spearman correlation co-efficient $(r)$ with quantification of the 
Table 1 Transcriptome dataset comparisons with HR RT-PCR and Haruna nijo fl cDNAs

\begin{tabular}{llll}
\hline Transcriptome Version & BaRTV1.0 & BaRTV1.0-QUASI & HORVU \\
\hline \# HR RT-PCR products & 220 & 220 & 191 \\
Pearson Correlation & 0.793 & 0.828 & 0.769 \\
Spearman Ranked Correlaton & 0.795 & 0.830 & 0.768 \\
\# Complete HN flcDNAs & 17,619 & 17,695 & 17,099 \\
\# Genes & 60,444 & 60,444 & 81,683 \\
\# Transcripts & 177,240 & 177,240 & 334,126 \\
\hline
\end{tabular}

AS events when compared to the HORVU dataset (Table 1). For the AS events detected in BaRTv1.0 and HORVU, we plotted the percentage spliced in (PSI) values (the fraction of mRNAs that represent the isoform that includes most exon sequence. The fraction of mRNAs with the most sequence spliced out (shortest transcript length) is not reported) from HR RT-PCR and RNA-seq for each of the three biological replicates from five different barley organ and tissue samples (giving 1992 and 1642 data points respectively) (Fig. 3a and b). Pearson and Spearman ranked correlation $(r)$ of the AS proportion values showed an improvement when comparing the HR RT-PCR with the three RNA-seq reference transcript datasets, HORVU (0.769 and 0.768), BaRTv1.0 (0.793 and 0.795) and BaRTv1.0-QUASI 0.828 and 0.83) (Table 1; Additional file 1: Table S4). We conclude that BaRTv1.0 (and the derived BaRTv1.0-QUASI) RTD is a comprehensive, nonredundant dataset suitable for differential gene expression and AS analyses.

\section{BaRTv1.0 genes and transcripts}

We next explored the characteristics of BaRTv1.0 genes and transcripts. A total of $57 \%$ of the BaRTv1.0 genes contained introns and had on average $\sim 7.7$ exons per gene (Table 2). Around $60 \%$ of the multi-exon genes had multiple transcripts supporting the occurrence of widespread AS in barley. Each transcript isoform within the dataset is unique based on splice site usage (containing at least one unique splice site). Analysis of the 177,240 predicted transcripts in BaRTv1.0 showed the expected distribution of canonical splice site dinucleotides. Of the 224,654 splice junctions examined, $98.2 \%$ of the introns spliced out have the expected GT..AG splice site dinucleotides, $1.7 \%$ had GC-AG dinucleotide borders, and $0.1 \%$ showed the U12- intron-dependent splicing AT-AC dinucleotide splice sites. Half of these splice junctions were observed in all the RNA-seq datasets tested but, $1.3 \%$ were unique to a single dataset, indicating unique tissue or condition specific splicing (Additional file 1: Table S5).

We then used the SUPPA software version 2.3 [1] to determine different splicing events and their frequency in our transcript dataset. We identified all the expected major forms of AS, including alternative $5^{\prime}$ and 3' splice site selection (Alt 5'ss; Alt 3'ss), exon skipping (ES) and intron retention (IR). Frequencies of the different AS events were consistent with studies in other plant

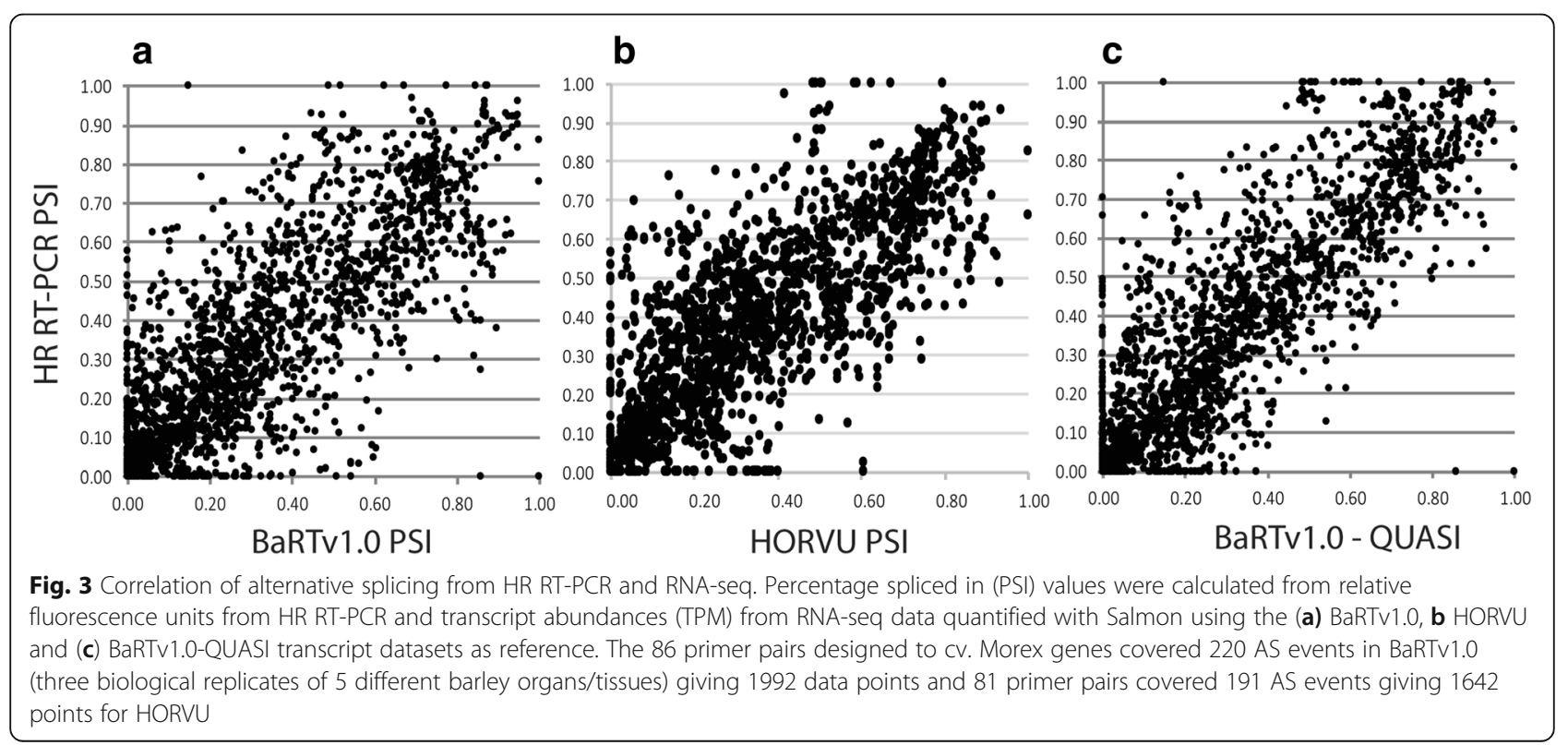


Table 2 Characteristics of barley genes and transcripts in BaRTv1.0. Percentages given are of total number of genes or transcripts

\begin{tabular}{ll}
\hline Number of genes & 60,444 \\
Number of predicted transcripts & 177,240 \\
Single exon genes & $25,719(43 \%)$ \\
Multi exon genes & $34,725(57 \%)$ \\
Single transcript genes & $39,534(65 \%)$ \\
Single exon transcripts & $27,754(16 \%)$ \\
Multi-Exon transcripts & $149,486(84 \%)$ \\
Number of Multi-exonic genes with alternative transcript variants & $20910(60 \%)$ \\
Mean number of transcripts per gene & 2.93 \\
Number of distinct exons & 466,247 \\
Mean number of distinct exons per gene & 7.7 \\
Mean transcript locus size (first to last exon) (nt) & 5,633 \\
Mean exon size (nt) & 573 \\
\hline
\end{tabular}

species (Alt 5' - 23.6\%; Alt 3' - 28.0\%; ES - 9.7\% and IR - 37.9\% - Table 3) [14, 34, 45]. Of the alternative 3' splice site events, 2743 were of the NAGNAG type where two alternative 3 ' splice sites are found 3 nt apart. Alternative NAGNAG 3' splice sites can be of functional importance and are commonly found in human and plant genomes in coding sequences where they can add or remove a single amino acid and may be subject to regulation $[8,48,49]$.

\section{Differential expression and differential alternative splicing in different barley organs/tissues}

The major motivation for developing BaRTv1.0 was to exploit the fast, alignment-free transcript quantification software, Salmon, which requires an RTD to quantify transcript isoform abundances using $\mathrm{k}$-mer indexing and counting [42]. We used RNA-seq data from three biological repeats of five organs/tissues of Morex to quantify transcripts with Salmon and BaRTv1.0-QUASI. Differential expression (DE) at both gene and transcript levels, differential AS (DAS) genes and differential transcript usage (DTU) were analysed using the recently developed 3D RNA-seq App [9, 10, 23]. All the parameters, underlying software, statistical tests used to generate the $\mathrm{DE}$ and DAS results for the 5 barley tissues are given in the supplementary 3D analysis report. We removed poorly expressed transcripts from the dataset by stringent filtering (transcripts with $\geq 1$ counts per million in at least 4 of the 15 samples were retained). A gene/transcript was significantly DE if it had an adjusted $p$-value of $<0.01$ and $\log _{2}$ fold change of $\geq 1$. To identify significant DAS genes, consistency of expression changes ( $\log _{2}$ fold change) between the gene and its transcripts was determined along with the change in splice ratio ( $\Delta$ Percent Spliced $-\Delta P S)$. A DAS gene had at least one transcript which differed significantly from the gene and with an adjusted p-value of $<0.01$ and had at least a $0.1(10 \%)$ change in $\triangle \mathrm{PS}$. Across the five organs and tissues, we detected expression of 60,807 transcripts from 25,940 genes. 20,972 genes were significantly DE across all tissues and 2791 genes showed significant DAS (Fig. 4a \& d; Additional file 1: Table S6). The overlap between DE and DAS genes (those genes regulated by both transcription and AS) was 2199 such that 592 genes were DAS-only and regulated only at the level of AS with no change in overall gene expression. We also identified 4151 transcripts with significant DTU which underpins the differential AS. DTU transcripts behave differently from other transcripts of DAS genes and were identified by testing the expression changes of every transcript against the weighted average of all the other

Table 3 Frequencies of different alternative splicing events in BaRTv1.0

\begin{tabular}{lll}
\hline Type of event & $\#$ & $\%$ \\
\hline${\text { Alternative } 3^{\prime}}^{\prime}$ & 44,590 & $28.0 \%$ \\
Alternative $5^{\prime}$ & 37,626 & $23.6 \%$ \\
Retained intron & 60,327 & $37.9 \%$ \\
Skipped exon & 15,387 & $9.7 \%$ \\
Mutually exclusive exons & 1,311 & $0.8 \%$ \\
& 159,241 & $100.0 \%$ \\
\hline
\end{tabular}


transcripts of the gene [10]. DTU transcripts differ significantly from the gene level and show a $\triangle \mathrm{PS}$ of $\geq 0.1$ with an adjusted p-value of $<0.01$. Pair-wise comparison of the number of up and down DE genes between each of the tissues showed that the two most related tissues (different developmental stages of inflorescence) had the fewest genes that were differentially expressed between them (ca. 700) but also had the highest number of DE genes when compared to other organs/tissues (ca. $14.5 \mathrm{k}$ between INF2 and NOD) (Fig. 4b). There were ca. 10-fold fewer genes showing differential AS and pair-wise comparisons, which again showed that the two inflorescence tissues had the fewest numbers of DAS genes between them and INF2 compared to NOD, EMB and LEA had the highest numbers of DAS genes (Fig. 4c). These results suggest that barley inflorescence transcriptomes differ substantially from shoot leaf, internode and embryos.

Hierarchical clustering of gene expression profiles of the 20,971 DE genes (DE-only and DE + DAS genes) across the organs/tissues identified clusters of genes that were co-ordinately and differentially expressed in each of the organs and tissues (Fig. 4d). Cluster $1(n=2435)$ contained genes that were most highly expressed in the embryo, cluster $3(n=2477)$ and $6(n=2714)$ in the internode, cluster $5(n=2498)$ and $8(n=4906)$ in inflorescences and cluster $4(n=1880)$ and $9(n=1316)$ in leaf (Fig. 4d; Additional file 1: Table S6). Hierarchical clustering also identified 2768 transcripts differentially expressed DTU that showed some specificity of expression in each of the sampled tissues (Fig. 4e; Additional file 1: Table S6). Cluster $1(n=292)$ contains DTUs that are up-regulated in the embryo, Cluster 4, 5 and 6 (total $n=885$ ) in the internode and cluster 7 (total $n=355$ ) in shoot leaf. Cluster $3(n=225)$ showed a cluster of DTU transcripts at the early stage of inflorescence development, cluster $8(n=296)$ at both stages of inflorescence development and cluster $9(n=559)$ at the later stage of inflorescence development. Some DTUs show significant isoform switching between the tissues and organs indicating an important role for AS in these genes (Additional file 2: Figure S5 for examples). Thus, extensive differential gene and transcript expression and differential alternative splicing was revealed among the different samples using BaRTv1.0.

\section{Validation of differential AS from RNA-seq with HR RT-PCR and RNA-seq}

To validate differential AS observed for individual genes among the different organs/tissues, we compared the RNA-seq quantifications of the 86 AS genes and 220 transcripts used in HR-RT-PCR. HR RT-PCR data showed over two-thirds of these transcripts had a significant differential AS ( $p=<0.001 ;>5 \%$ change) across the five samples (Additional file 1: Table S7). Given the

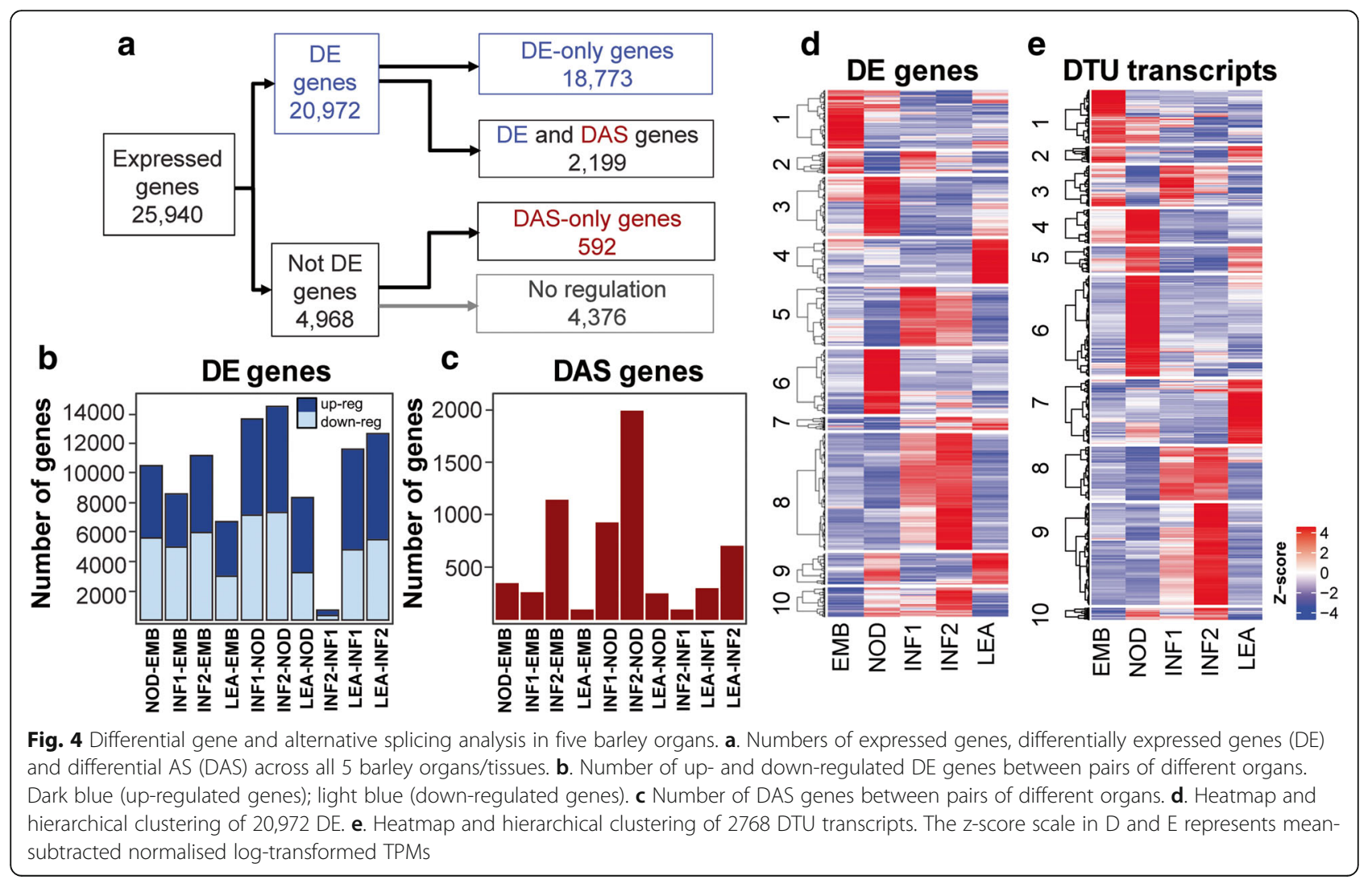


RNA samples used in both the HR RT-PCR and RNAseq was the same, we were able to directly compare differential AS observed at the individual gene level. For example, primer pairs Hv110 (HORVU5Hr1G027080; BART1_0-u34104) and Hv118 (HORVU1Hr1G078110; BART1_0-u5387) assay AS events that generate two alternative transcripts in BaRTv1.0. The AS transcripts are the result of alternative $5^{\prime}$ splice sites, $5 \mathrm{nt}$ (Fig. 5a) and $4 \mathrm{nt}$ (Fig. 5b) apart respectively. In each case selection of the distal $5^{\prime}$ splice sites produce the full-length CDS and use of the proximal $5^{\prime}$ splice site will result in a frame-shift and premature termination codons. Primer pair Hv173 (HORVU7Hr1G062930; BART1_0-u52907) assays alternative selection of two $3^{\prime}$ splice sites 33 nt apart (Fig. 5c) and Hv217 (HORVU7Hr1G071060; BART1_0-u52404) assays retention of intron 1 (Fig. 5d). Each of these examples show the pattern of AS across the tissues are essentially equivalent between HR RT-PCR and RNA-seq (Fig. 5) and overall, we observed remarkable consistency. Thus, there is good agreement between the differential alternative splicing analysis from the RNA-seq data and the experimental verification with HR RT-PCR. These data provide strong support for the value of using BaRTv1.0 and BaRTv1.0-
QUASI as reference datasets for accurate expression and AS analysis.

\section{Complex patterns of AS}

A principal aim of establishing BaRTv1.0 was to achieve higher accuracy of differential expression and AS analysis in barley RNA-seq datasets by improved transcript quantification. While the overall number of Morex transcripts in the HORVU collection (ca. $344 \mathrm{k}$ ) was approximately halved in BaRTv1.0 (ca. $177 \mathrm{k}$ ) (Table 1), some genes have multiple transcripts due to combinations of complex AS events. To fully characterise multiple gene transcripts will require long read transcript sequencing to determine the different combinations of splice sites used. However, we can determine the selection of multiple alternative splice sites using HR RT-PCR. As an example, AS events in BART1_0-u51812, which codes for a WW domain-containing protein, were validated. BART1_0-u51812 contains 44 different transcript isoforms in the BaRTv1.0 dataset due to unique combinations of different AS events (Fig. 6a). We analysed two regions that showed complex AS: between exons 2 and 3 and between exons 6 and 7 by HR RT-PCR (Fig. 6). HR a Hv110 - clathrin adaptor complex subunit
BART1_0-u34104; HORVU5Hr1G027080

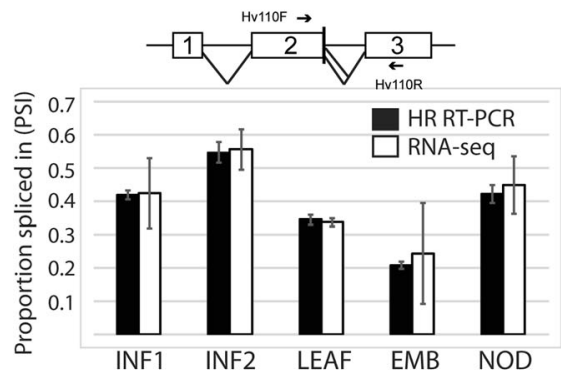

C

Hv173-CYP38; cyclophilin 38 BART1_0-u52907; HORVU7Hr1G062930
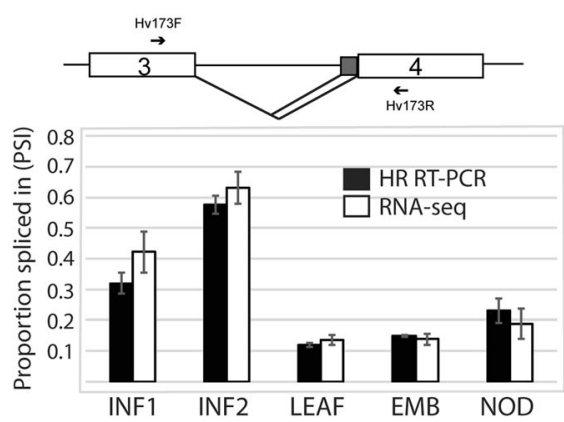

b Hv118-S-adenosyl-L-methionine-dependent methyltransferases superfamily protein BART1_0-u05387; HORVU1Hr1G078110

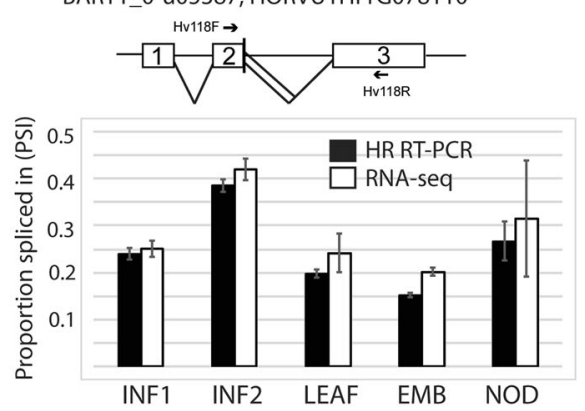

d

Hv217- AtSPO11-2; DNA topoisomerase VIA subunit BART1_0-u53404; HORVU7Hr1G071060

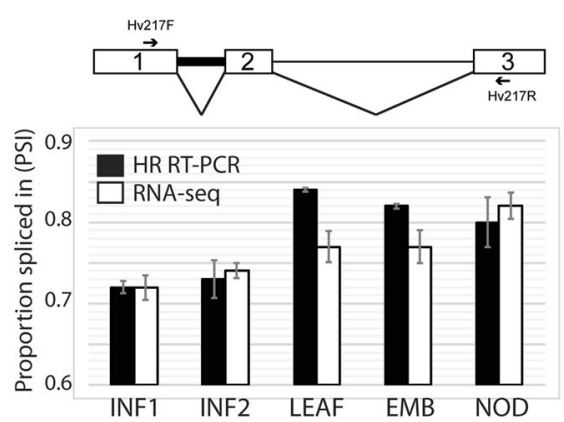

Fig. 5 Comparison of alternative splicing in different barley tissues with HR RT-PCR and RNA-seq data. Splicing proportions of four different genes in 5 different barley tissues are presented. a. Hv110; HORVU5Hr1G027080, b. Hv118; HORVU1Hr1G078110, c. Hv173; HORVU7Hr1G062930, d. Hv217; HORVU7Hr1G071060. Schematic transcript/AS models are presented above histograms of PSls derived from HR-RT-PCR (black) and RNA-seq (white) with standard error bars across three biological repeats. White boxes - exons, lines - introns; chevrons - splicing events; grey boxes region between alternative splice sites; thick intron line represents an intron retention 
RT-PCR analysis identified fully spliced (FS), two alternative $5^{\prime}$ splice sites and retention of intron 2 as the main AS events between exons 2 and 3. In addition, four minor HR RT-PCR products were also identified and these were characterised as two further alternative 5' splice sites and two alternative exons from the BaRTv1.0 transcripts (Fig. 6b). Between exons 6 and 7, the main AS events are fully spliced, retention of intron 6, inclusion of an alternative exon and an alternative $5^{\prime}$ splice site (Fig. 6c). HR RT-PCR across exons 6-7 (primer pair Hv79 in exons 6 and 8) accurately identified these AS events (Fig. 6C). These AS events were also quantified using transcript abundances from the RNA-seq data using BaRTv1.0_QUASI and showed good agreement with the HR RT-PCR results with Pearson correlations of 0.92 for the Hv78 regions and 0.73 for the Hv79 region. These examples support the accuracy of alternative splicing found in BaRTv1.0 and that the proportions of alternative splice sites selected in short-read RNA-seq can be determined.

\section{Discussion}

Comprehensive reference transcript datasets are required for accurate quantification of transcripts for expression analysis using RNA-seq. Quantification at the transcript level improves gene level expression estimates and allows robust and routine analysis of alternative splicing. Here we describe the BaRTv1.0 transcript dataset or transcriptome for barley, produced by merging and filtering transcripts assembled from extensive RNA-seq data and its utility in differential expression and differential alternative splicing. The transcripts were assembled against cv. Morex and this reference transcript dataset is therefore a Morex assembly. BaRTv1.0 achieves a balance between maximising transcript diversity - all 177,240 transcripts have a unique combination of different splice sites with strong junction support - and reducing the numbers of misassembled transcripts, transcript fragments and redundant transcripts. This barley transcript dataset represents the first stage of an evolving resource which will continue to improve and expand as more complete barley genomes are released and by incorporation of new Illumina short read data along with single molecule sequencing (Pacific Biosciences or Oxford Nanopore Technology) datasets when they become available. Long-read data will confirm transcript features proposed by the short-read assemblies by defining the exact combinations of different AS events and 5 ' and 3 ' ends and may identify rare transcripts. The transcript and splice junction data generated here will be valuable in improving the barley genome annotation. Finally, the BaRTv1.0 transcript dataset will enable accurate gene and transcript level expression and AS analysis increasing our understanding of the full impact of AS and how transcriptional and AS regulation of expression interact to determine barley development, responses to environment and ultimately important crop phenotypes such as yield, disease resistance and stress tolerance.

BaRTv1.0 represents 60,444 genes, which is considerably fewer than the 81,683 genes reported in the current barley genome [35] where residual gene fragmentation has likely inflated the number of annotated genes. However, the arrangement of BaRTv1.0 transcripts have identified mis-annotated chimeric genes in the barley reference genome, helping to improve gene resolution. BaRTv1.0 was established using RNA-seq data containing approximately 19 billion reads from a range of different biological samples (organs, tissues, treatments and genotypes) and was assembled initially against the Morex genome. The sequence depth and rigorous filtering and validation allowed us to establish a diverse set of highquality, robust and experimentally supported transcripts.

A key function of the BaRTv1.0 transcript dataset is improved accuracy of transcript abundance. Variation in the $5^{\prime}$ and $3^{\prime}$ ends of transcripts of the same gene was shown previously to affect transcript quantification in Arabidopsis [61] and similar results for 3' end variation have been found in human RNA-seq analysis [53]. Extending the sequences of shorter transcripts with genomic sequences such that all transcripts of a gene had the same $5^{\prime}$ and 3 ' ends improved the accuracy of transcript quantification compared to experimental data [61]. We also found an improvement in the quantification of transcripts and splicing proportions by applying the same approach to produce the BaRTv1.0-QUASI version, specifically for quantification of alternatively spliced isoforms (Table 1). The continued development of reference transcript datasets for other lines and cultivars will be essential for accurate gene expression and AS analysis. One significant application will be to enable genome-wide association studies using gene expression data to identify eQTLs and transcript abundance/splicing ratios to identify splicing QTLs [56].

To demonstrate the value of the new RTD for gene expression studies and AS analysis, we used BaRTv1.0QUASI to quantify transcripts in the five developmental organs and tissues RNA-seq datasets that we had used previously for HR RT-PCR optimisation and validation. We observed extensive differences in gene expression and AS among the five divergent samples. Clustered coexpression patterns clearly showed that the different organs and tissues have distinct transcriptomes reflecting major differences in both transcription and AS, as recently demonstrated in the cold response in Arabidopsis [10]. The abundance of individual BaRT transcripts in these five organs/tissues, and in the eleven other organs and tissues used to annotate the barley genome [35] are displayed in a barley reference transcript database website https://ics.hutton.ac.uk/barleyrtd/index.html. 


\section{a BaRTv1_0-u51812; HORVU7Hr1G044850 - WW containing protein}

Exon Structure of BART1_0-u51812

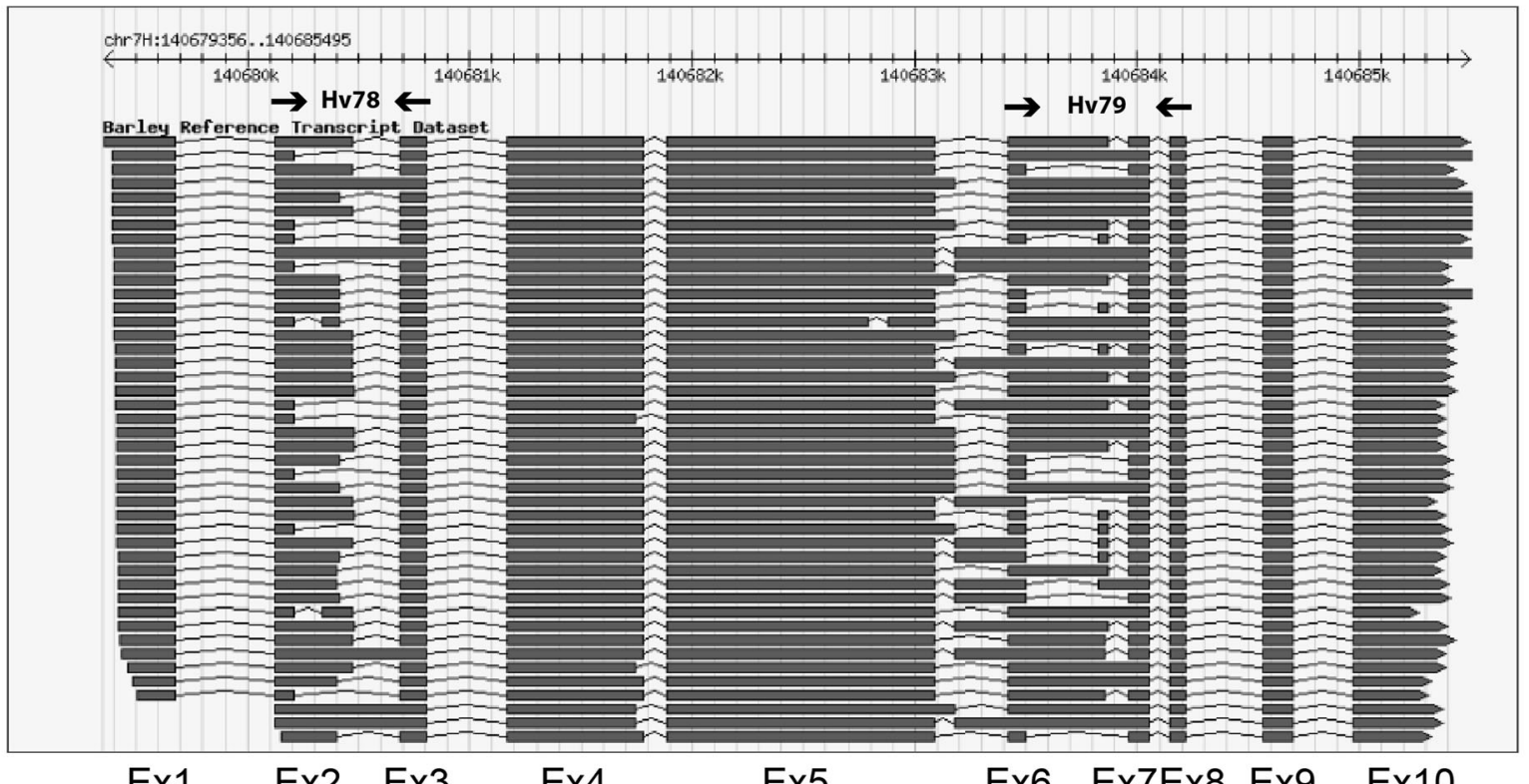

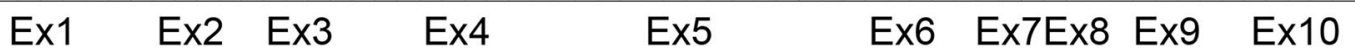

b
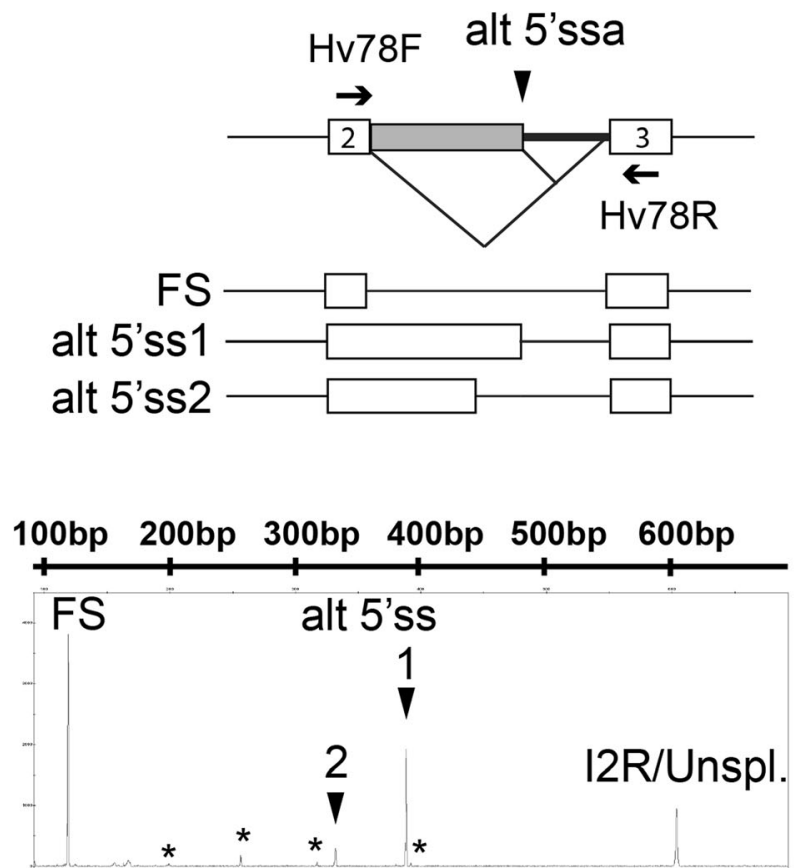

C
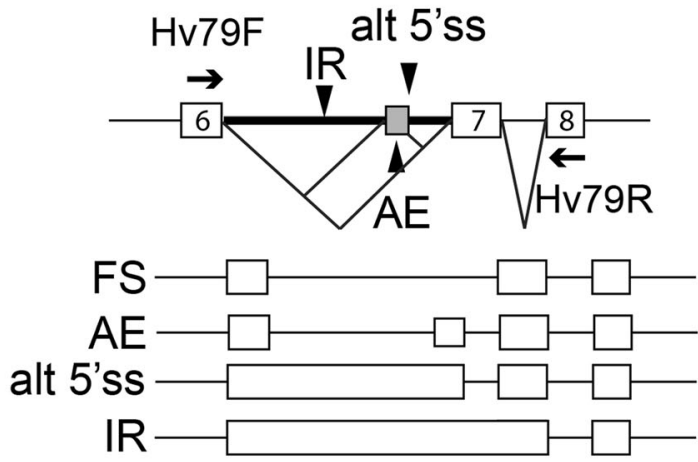

100bp 200bp 300bp 400bp 500bp 600bp 700bp

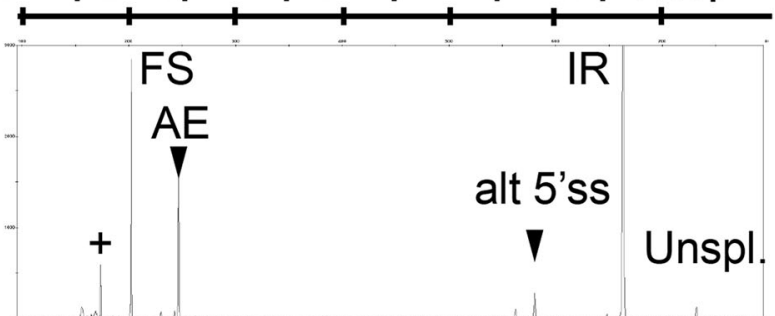

Fig. 6 Alternative splicing in a WW domain containing protein gene (BART1_0-u51812). a. BART1_0-u51812 transcript models represented in the BaRTv1.0 database. $\mathbf{b}$. AS events involving intron 2 validated by HR-RT-PCR. c. AS events between exon 6 and 8 validated by HR-RT-PCR. Electropherogram output from the ABI3730 shows the HR RT-PCR products ( $x$-axis RT-PCR products (bp); $y$-axis relative fluorescence units). The products expected from RNA-seq are indicated as FS - Fully spliced, AE - Alternative exon, Alt 5'ss - Alternative 5' splice site, IR-intron retention and Unspl.-Unspliced. * in B. indicates minor alternative transcripts identified in HR RT-PCR and in RNA-seq. + in C. indicates an uncharacterised alternative transcript identified in HR RT-PCR 
Barley is adapted to a wide range of environments and is grown for many purposes. As a result, different cultivars/genotypes will have unique transcriptome profiles that will respond differently to varying developmental or environmental conditions and challenges. BaRTv1.0 enables rapid and robust analysis of gene expression and AS in a wide range of experimental scenarios. BaRTv1.0 is based on cv. Morex but used RNA-seq data from a wide-range of cultivars and lines. We anticipate significant and incremental improvements in subsequent BaRT iterations by adding new short and long-read RNA-seq datasets but understand the need to capture the diversity of different transcripts which will occur among different cultivars and landraces. Sequence variation among different lines will generate quantitative variation in expression and alternative splicing [21]. Therefore, using the methods presented here, RTDs for other widely used cultivars can be generated. For example, construction of RTDs for Golden Promise (used for genetic transformation studies) [38], Bowman (the background cultivar for a collection of near isogenic lines) [15] and Barke (a cultivar more relevant to modern European cultivated barley) [44] would all have specific utility. Ultimately, transcript data from a wide range of genotypes will stimulate the move towards the development of a reference pantranscriptome to parallel the generation of the barley pangenome sequence.

\section{Conclusions}

A comprehensive, non-redundant barley reference transcript dataset called BaRTv1.0 has been generated, which enables fast, precise transcript abundances. Downstream analysis of transcript abundances in five barley organs/ tissues, identified significant differential expression of many genes and transcripts. BaRTv1.0 is part of a unique pipeline that facilitates the robust routine analysis of barley gene expression and AS. The reference transcripts have broader opportunities to develop unique expression markers, support proteomic resources for barley and enable transcript/co-expression/regulatory networks. The pipeline developed here has relevance to the development of other crop reference transcript datasets.

\section{Materials and methods}

An experimental and bioinformatics workflow showing the assembly, filtering and validation approach taken is shown in Fig. 1.

\section{Selected RNA-seq datasets and data processing}

A total of 11 large RNA-seq datasets consisting of 808 samples including replicates, were selected to assemble a barley transcriptome (Additional file 1: Table S1). Eight publicly available datasets were downloaded from NCBI - Sequence Read Archive database (https://www.ncbi. nlm.nih.gov/sra/) and the 3 remaining datasets are currently unpublished. All datasets were produced using Illumina platforms and were selected based on being the most recent datasets with the longest read length available (mostly $>90 \mathrm{bp}$ and paired-end reads) with a quality of $q>=20$. All raw data were processed using Trimmomatic0.30 [5] using default settings to preserve a minimum Phred score of Q20 over $60 \mathrm{bp}$. One of the samples (NOD1) was over-represented with respect to read numbers due to a repeat run being necessary, and was therefore subsampled to 60 million reads. Read quality before and after trimming was performed using FastQC (fastqc v0.11.5) (https://www.bioinformatics.babraham.ac.uk/projects/fastqc/).

\section{Transcriptome assembly Alignment}

Transcript assembly was performed using a data pipeline that initially used STAR (version 2.5 [18];) to align reads from each of the 808 samples individually to the latest barley cv. Morex reference genome (version 160404_barley_pseudomolecules_parts_masked /Hv_IBSC_PGSB_v2) [35]. Many alignment programmes use a two-step approach to identify exon junctions and then use the junctions to guide the final alignment [20]. A three-step STAR alignment approach was developed to improve alignment accuracy and identification of splice junctions and to take into consideration the sequence variation in reads from different cultivars and lines used. This approach further captured splice junctions from tissue/conditions samples where the amount of material or sequencing depth were limited or where genotypes were represented by small numbers of samples. In the first pass, reads were mapped to the genome allowing a single mismatch and only those with an overhang minimum of $10 \mathrm{bp}$ on each side of the splice junction were taken forward. This step identified 1, 057,650 splice junctions, many of which were supported by only a single read. These splice junctions with 5 or more uniquely mapped reads (reads that match only one genomic position) were kept. In some cases, reads align equally to several genomic locations, in these cases only splice junctions containing at least 10 (multi-mapped) reads were kept. After filtering, the remaining 206,688 splice junctions were used as annotation for the second pass. In the second pass the alignment was relaxed to allow 2 mismatches in the splice junction region with an overhang minimum of $7 \mathrm{bp}$. This step identified 1,088,440 splice junctions and these were further filtered to select splice junctions on the basis of one of three sets of criteria depending on the range of expression levels between the different samples: a) splice junctions with 3 or more uniquely mapped reads ( 5 or more reads if multi-mapped reads are present) in at least 2 samples; b) splice junctions 
with 2 or more uniquely mapped reads in at least 5 samples or c) splice junctions supported by 1 or more uniquely mapped reads in at least 10 samples and allowing for $2 \%$ mismatches in the alignment of reads outside the splice junction. In the final pass, the 323,619 filtered splice junctions from the previous step were used as annotation and no new splice junctions were allowed. In this step, the read mismatch rate was relaxed to $3 \%$ to allow more reads to map. In all three passes, only canonical splice junctions (GT..AG, GC..AG and AT..AC) and concordant alignments were kept.

\section{Transcript assembly}

After STAR alignment, each sample was run individually using StringTie (version 1.3.3b) [43]. Different combinations of StringTie parameters were extensively tested and the parameters that produced the best assembly were retained (see Results). Evaluation of each assembly was performed based on comparison with HR RT-PCR data consisting of 86 genes and 220 alternatively spliced RT-PCR products (see Results). To evaluate the completeness of the transcripts assembled, 22,651 Haruna nijo fl-cDNAs [37] were aligned using BLASTn (blastn, version ncbi-blast-2.2.28+ [2];) to each RNA-seq transcriptome assembly generated. All fl-cDNAs with $\geq 90 \%$ coverage and $\geq 90 \%$ identity were identified and the total number was considered a measure of completeness. Optimal StringTie parameters were coverage $(-c 2.5)$; gap between readings triggering a new bundle (-g 50); isoform fraction was set at $-\mathrm{f} 0$, gene abundance estimation was set as output (-A), minimum anchor length for junctions 5 $(-\mathrm{a})$; minimum junction coverage $0.1(-\mathrm{j})$ and fraction of bundle allowed to be covered by multi-hit reads $1(-M)$.

\section{Removal of low abundance transcripts}

Salmon is a software tool that utilises a defined set of reference sequences to perform a rapid, alignment-free estimation of isoform abundances by using $\mathrm{k}$-mer indexing and counting. It uses an accelerated expectation-maximization algorithm for quantifying isoform abundance, which is given in transcripts per million (TPM). All 808 individual StringTie assemblies were merged with StringTie-merge, after all 808 read samples were aligned to the merged reference transcriptome with Salmon (version Salmon-0.8.2) [42] to obtain transcript quantification. All transcripts that were expressed at less than 0.3 TPM, across all samples, were filtered out.

\section{Assembly merge}

All 808 assembly predictions from StringTie were merged using StringTie-merge to create a unique consensus assembly version. A minimum isoform fraction of $0(-\mathrm{f})$ and a minimum input transcript TPM of $0.1(-\mathrm{T})$ was used in
StringTie-merge. The consensus transcriptome, after filtering out the transcripts less than 0.3 TPM, was further merged (gtf format) with the 22,651 Haruna nijo (HN) fl cDNAs [37]. The HN fl cDNAs were previously mapped to the barley cv. Morex genome with the GMAP tool (version 2017-10-30) [58]. Finally, we used TransDecoder (version 5.3.0) [24] and BLASTp to identify and filter out all transcripts equal to or less than $300 \mathrm{bp}$ (8831 transcripts) with less than $70 \%$ of coverage and identity protein homology with the protein datasets from 3 reference Poaceae species - Oriza sativa (v7_JGI), Brachypodium distachyon (Bd21-3 v1.1) and Sorghum bicolor (v3.1.1) (https://genome.jgi.doe.gov/portal/) (Additional file 2: Figure S4) to establish BaRTv1.0.

\section{Alternative splicing analysis}

The newly created non-redundant BaRTv1.0 consensus transcriptome was further refined to allow accurate quantification of AS as described previously, to create a separate dataset specifically for quantification of AS isoforms (BaRTv1.0 - QUASI) [61]. All transcripts with shorter $5^{\prime}$ and $3^{\prime}$ UTR regions were padded out to the $5^{\prime}$ and $3^{\prime}$ ends of the longest transcript of that gene using the $\mathrm{cv}$. Morex genome.

\section{High resolution RT-PCR}

The RNA from five of sixteen developmental stages of barley cv. Morex was used for HR RT-PCR validation [35]. This consisted of three biological replicates of leaf tissue (LEA) sampled from seedlings at 17 days after planting (dap); the third stem internode (NOD) dissected at 42 dap; whole developing inflorescence tissue sampled at 30 dap (INF1) and 50 dap (INF2) and embryonic tissue (including mesocotyl and seminal roots; EMB) dissected after 4 days. High resolution RT-PCR was performed essentially as described previously [51]. A panel of 86 primer pairs covering 220 RT-PCR products (Additional file 1: Table S3), were designed to barley genes that showed evidence of AS and more than 100 RNA-seq reads for each primer pair to support transcription, with the exception of 14 primer pairs numbered between primers \#14 and 51. These primers were designed to genes already under study and consisted of splicing factor genes, clock response genes and Rubisco activase (Additional file 1: Table S3). Primers were designed to amplify products between 100 and $700 \mathrm{bp}$ to capture the different splicing events. The $5^{\prime}$ upstream primer was $5^{\prime}$ labelled with 6-Carboxyfluorescein (6-FAM). Total RNA $(5 \mu \mathrm{g})$ was used for first-strand cDNA synthesis by reverse transcription with oligo (dT)18 using Ready-To-Go YouPrime First-Strand Beads (GE Healthcare) in a final volume of $20 \mu \mathrm{L}$. RT-PCR was performed as described [51] and the resultant RT-PCR products representing AS transcripts were detected on an ABI3730 DNA Analyzer (Thermo 
Fisher Scientific) along with GeneScan 500 LIZ size standard (Applied Biosystems). RT-PCR products were accurately sized and peak areas calculated (Relative Fluorescence Units - RFUs) using GeneMapper (ABI) software.

\section{Comparing HR RT-PCR and RNA-seq alternative splicing proportions}

To assess the accuracy of BaRTv1.0 to detect changes in AS in the RNA-seq data, we compared the splicing proportions for AS events from HR RT-PCR with those calculated from the RNA-seq data using the HORVU transcript set, BaRTv1.0 and BaRTv1.0-QUASI as transcript references. To establish the correlations, a number of considerations were required. First, HR RT-PCR data reports exclusively on the events that occur within a gene bordered by the primers used for the analysis. The RNA-seq data reports on individual transcripts that may contain multiple AS events or have an alternative transcript start and/or stop. For this reason, multiple RNAseq transcripts may represent the same AS product that is detected by HR RT-PCR. We therefore developed a method (https://github.com/PauloFlores/RNA-Seq-validation) that determined the size of the expected PCR product by aligning the primer pairs against each RNAseq transcript and determining the predicted length that PCR would produce. The TPM values of all transcripts that produce the same AS PCR product were added together to give a combined RNA-seq value for that PCR product. The proportions of the different AS products for both HR-RT-PCR and RNA-seq were then subsequently calculated and correlated.

Firstly, the method mapped the HR RT-PCR primers to the transcriptome using BLAST (blastn-short command; version ncbi-blast-2.2.28+ [2];). All transcripts with perfect identity and coverage for both reverse and forward primers at one gene transcript location were selected (http://ics.hutton.ac.uk/barleyrtd/primer_list.html). Secondly, the distance was calculated between the pairs of primers for each selected transcript, and thirdly, transcripts with equal product length associated with the same pair of primers were clustered together. Fourthly, five reference samples from the sample dataset, each with 3 biological replicates to give 15 datasets [26] were individually quantified by Salmon (version Salmon-0.8.2 [42];). The five reference samples consisted of 4-day old embryos dissected from germinating grains (EMB), young developing inflorescences $(5 \mathrm{~mm})$ (INF1), developing inflorescences $(1-1.5 \mathrm{~cm})$ (INF2), developing tillers at 6 leaf stage, third internode (NOD) and shoots from seedlings (LEA). The levels of expression (in TPM) from Salmon were summed for transcripts with the same RT-PCR product lengths. For each pair of primers and allowing for a difference of \pm 6 bp (to allow for inaccuracies in HR RT-PCR size calling), products of the same length between HR RT-
PCR and RNA-seq were identified. Finally, based on the calculated values of RNA-seq levels of expression and the calculated values of HR RT-PCR for each RT-PCR product, the proportions of the alternative transcripts were calculated. The proportions determined the level of one gene transcript in relation to all the alternative transcripts. It was calculated by dividing a transcript TPM (RNA-seq) or RFU (HR RT-PCR) value by the total number of transcript values for a particular gene. Pearson's and Spearman's correlation co-efficient $(r)$ was calculated to determine the extent to which the AS proportions fluctuate between the RNA-seq and HR RT-PCR methods. (see Additional file 2: Figure $\mathrm{S} 6$ for a pipeline summary).

\section{Percent spliced in values and identification of alternative splicing type}

SUPPA version 2.3 [1] determined AS events and calculated the relative inclusion values of AS events. Outputs from Salmon were fed into SUPPA to quantify AS events across the tissue sample datasets and generate percentage spliced in (PSI) values.

\section{Generation of the BaRTv1.0 database}

A database and website front-end were constructed to allow easy access to BaRTv1.0 transcripts and expression analyses using the LAMP configuration (Linux, Apache, mySQL, and Perl). Additional annotation was added to the transcripts by homology searching against the predicted peptides from rice (rice pseudo-peptides v 6.0; [40]) and from Arabidopsis thaliana (TAIR pseudopeptides v 10, The Arabidopsis Information Resource) using BLASTX at an e-value cutoff of less than 1e-50 [2]. The website https://ics.hutton.ac.uk/barleyrtd/index.html allows users to interrogate data through an entry point via three methods: (i) a BLAST search of the reference barley assembly or the predicted transcripts; (ii) a keyword search of the derived rice and Arabidopsis thaliana BLAST annotation, and; (iii) a direct string search using the transcript, gene, or contig identifiers. To distinguish this new set of predicted genes and transcripts from previously published 'MLOC_' and HORVU identifiers, they have subsequently been assigned a prefix of 'BART1_0-u00000' for the unpadded or 'BART1_0-p00000' for the padded QUASI version, with BART1_0-p00000.000 representing the individual transcript number.

The RNA-seq TPM values for the developmental stages of barley (Morex cultivar) [35] at the replicate and stage are shown in both graphic and tabular formats for each gene. The exon structures of the transcripts for each gene are shown in graphical form, and links to the transcripts themselves provides access to the transcript sequences in FASTA format. Each transcript has also been compared to the published set of predicted genes (HORVUs) in order to provide backwards compatibility. 


\section{Statistical analysis HR RT-PCR ANOVA}

Pairwise significance of the variation between the developmental tissues was assessed by analysis of variance (ANOVA). Each peak of each primer was analysed separately with three replicate values for each treatment combination. Response was measured as the percentage contribution of a particular isoform to the total transcripts measured, and ANOVA was carried out after an angular (arcsin) transformation was used to transform values from $[0,1]$ to $[-\pi / 2,+\pi / 2]$ to give the data a normal distribution [52]. ANOVA was conducted in $\mathrm{R}$ by using the code anova $(\operatorname{lm}(\mathrm{x} \sim$ tissue $))$ where $\mathrm{x}$ was the transformed splicing proportion for a site. Fisher's Least Significant Difference (LSD) test was performed for the pairwise comparisons between the different tissues tested at a p- value $<0.001$. In the subsequent analysis, we focused on those transcripts which showed a significant increase or decrease with a $5 \%$ difference between the means of the different plant tissues. This level of difference was selected because we previously determined that when comparing variation in technical reps in the AS RT-PCR system, the majority of transcripts showed a standard error of the mean of $<3 \%[29,51]$.

\section{Supplementary information}

Supplementary information accompanies this paper at https://doi.org/10. 1186/s12864-019-6243-7.

Additional file 1: Table S1. RNA-seq datasets from both public databases and in house unpublished data. Table S2. StringTie Parameters used in the different assemblies (sorted by transcript number). Table S3. Mean proportions of alternatively spliced products by HR-RT-PCR analyisis. Table S4. Correlation of HR RT-PCR data with BaRTV1.0, BaRTV1.0- QUASI and HORVU transcripts. Table S5. Splice Junctions and intron lengths. Table S6. Differentially expressed gene clusters and differential transcript usage gene clusters. Table S7. Pairwise significant changes in alternatively spliced transcripts detected by HR RT-PCR between different organs.

Additional file 2: Figure S1. 3 Pass Star alignment details. Figure S2. GMAP parameter options to align the 22,651 Haruna Nijo full-length cDNAs to the barley genome. Figure S3. Optimal StringTie assembly. Figure S4. Transdecoder annotation and filtering short transcripts. Figure S5. Examples of significant alternative transcript switches between developing inflorescence (INF1) and leaf (LEA) samples. Figure S6. Pipeline describing the algorithm to compare HR-RT-PCR and RNA-seq alternatively spliced transcript proportions and correlations.

\section{Acknowledgements}

We further acknowledge those involved with the ERC project 669182

'SHUFFLE' project in generating samples.

\section{Authors' contributions}

PR-F, MB, CS, C-DM, and RW conceived and designed the experiment and AS analysis. PR-F and C-DM performed the statistical and correlation analyses. PEH and JM prepared the RNA samples for the HR RT-PCR analysis and COordinated the sequencing. JF, JWSB, CS RW, AB, MS, CH, JK, SM, MCC and MZ generated RNA and libraries for RNA-seq. PR-F and MB assembled the RNAseq data. JF, GS and CS identified the AS genes and performed the HR RTPCR screening and analysis of the data. LM established the searchable database. PR-F, C-DM, JWSB, RZ, WG and CS performed the detailed analysis of the RNA-seq and HR RT-PCR data. CS, PR-F, RW and JWSB wrote the paper. All authors read and approved the final manuscript.

\section{Funding}

This research was supported and developed by Scottish Government Rural and Environment Science and Analytical Services division (RESAS) and funding from the Biotechnology and Biological Sciences Research Council (BBSRC) (BB/I00663X/1: A draft sequence of the barley genome, to RW). RNAseq datasets were produced through funding to $\mathrm{CH}$ through the BBSRC Sustainable Bioenergy Centre initiative grant no. BB/G016232/1, BBSRC Response Mode Grant No. BB/K01613X/1 to SM and RW and ERC project 669182 'SHUFFLE' to RW.

\section{Availability of data and materials}

BaRTV1.0 and BaRTV1.0 - QUASI are available as .fasta and. GFF files and can be downloaded from https://ics.hutton.ac.uk/barleyrtd/downloads.html. An additional version of the RTD is available in the Zenodo repository (https:// doi.org/10.5281/zenodo.3360434).

To develop BaRTV1.0 we used publicly available sequences from the Sequence Read Archive (SRA) or European Nucleotide Archive (ENA) (accession numbers: PRJEB13621; PRJEB18276; PRJNA324116; PRJEB12540; PRJEB8748; PRJNA275710; PRJEB14349; PRJEB19243). Further sequences were deposited in SRA or ENA (accession numbers PRJEB32063; PRJNA558196; PRJEB33952) and are in preparation for publication. These three datasets were used to increase coverage and splice junction confidence during the assembly of the barley RTD transcripts and are available from the corresponding author on reasonable request. Information on these datasets can be found in Additional file 1: Table S1.

Ethics approval and consent to participate

Not applicable

\section{Consent for publication}

Not applicable

\section{Competing interests}

The authors declare that they have no competing interests.

\section{Author details}

'Information and Computational Sciences, James Hutton Institute, Invergowrie, Dundee DD2 5DA, UK. 'Biomathematics and Statistics Scotland, Aberdeen AB25 2ZD, UK. ${ }^{3}$ Cell and Molecular Sciences, The James Hutton Institute, Invergowrie, Dundee DD2 5DA, UK. ${ }^{4}$ Division of Plant Sciences, School of Life Sciences, University of Dundee at the James Hutton Institute, Dundee DD2 5DA, UK. ${ }^{5}$ Present address: Institute of Biological, Environmental and Rural Sciences, Aberystwyth University, Gogerddan, Aberystwyth, Ceredigion SY23 3EB, UK. 'Present Address: MRC Protein Phosphorylation and Ubiquitylation Unit, Sir James Black Centre, School of Life Sciences, University of Dundee, Dundee DD1 5EH, UK.

Received: 6 June 2019 Accepted: 29 October 2019

Published online: 11 December 2019

\section{References}

1. Alamancos GP, Pagès A, Trincado JL, Bellora N, Eyras E. Leveraging transcript quantification for fast computation of alternative splicing profiles. RNA. 2015;21:1521-31. 
2. Altschul SF, Gish W, Miller W, Myers EW, Lipman DJ. Basic local alignment search tool. J Mol Biol. 1990;215:403-10.

3. Ashoub A, Müller N, Jiménez-Gómez JM, Brüggemann W. Prominent alterations of wild barley leaf transcriptome in response to individual and combined drought acclimation and heat shock conditions. Physiol Plant. 2018;163:18-29.

4. Bazin J, Romero N, Rigo R, Charon C, Blein T, Ariel F, Crespi M. Nuclear speckle RNA binding proteins remodel alternative splicing and the noncoding Arabidopsis Transcriptome to regulate a cross-talk between Auxin and immune responses. Front Plant Sci. 2018;9:1209.

5. Bolger AM, Lohse M, Usadel B. Trimmomatic: a flexible trimmer for Illumina sequence data. Bioinformatics. 2014;30:2114-20.

6. Bray NL, Pimentel H, Melsted P, Pachter L. Near-optimal probabilistic RNAseq quantification. Nat Biotechnol. 2016;34:525-7.

7. Brown JWS, Calixto CP, Zhang R. High-quality reference transcript datasets hold the key to transcript-specific RNA-sequencing analysis in plants. New Phytol. 2017;213:525-30.

8. Busch $\mathrm{A}$, Hertel $\mathrm{K}$. Extensive regulation of NAGNAG alternative splicing: new tricks for the spliceosome? Genome Biol. 2012;13:143.

9. Calixto CPG, Tzioutziou NA, James AB, Hornyik C, Guo W, Zhang R, Nimmo HG, Brown JWS. Cold-dependent expression and alternative splicing of Arabidopsis long non-coding RNAs. Front Plant Sci. 2019;10:235.

10. Calixto CPG, Guo W, James AB, Tzioutziou NA, Entizne JC, Panter PE, Knight $H$, Nimmo HG, Zhang R, Brown JWS. Rapid and dynamic alternative splicing impacts the Arabidopsis cold response Transcriptome. Plant Cell. 2018; 30:1424-44.

11. Calixto CPG, Simpson CG, Waugh R, Brown JWS. Alternative splicing of barley clock genes in response to low temperature. PLoS One. 2016;11: e0168028.

12. Capovilla G, Pajoro A, Immink RG, Schmid M. Role of alternative pre-mRNA splicing in temperature signaling. Curr Opin Plant Biol. 2015;27:97-103.

13. Carvalho RF, Feijão CV, Duque P. On the physiological significance of alternative splicing events in higher plants. Protoplasma. 2013;250:639-50.

14. Chamala S, Feng G, Chavarro C, Barbazuk WB. Genome-wide identification of evolutionarily conserved alternative splicing events in flowering plants. Front Bioeng Biotechnol. 2015;3:33.

15. Dahleen LS, Vander Wal LJ, Franckowiak JD. Characterization and molecular mapping of genes determining semidwarfism in barley. J Hered. 2005;96:654-62.

16. Dawson IK, Russell J, Powell W, Steffenson B, Thomas WT, Waugh R. Barley: a translational model for adaptation to climate change. New Phytol. 2015; 206:913-31.

17. Dobin A, Gingeras TR. Optimizing RNA-Seq mapping with STAR. Methods Mol Biol. 2016;1415:245-62.

18. Dobin A, Davis CA, Schlesinger F, Drenkow J, Zaleski C, Jha S, Batut P, Chaisson M, Gingeras TR. STAR: ultrafast universal RNA-seq aligner. Bioinformatics. 2013;29:15-21.

19. Filichkin S, Priest HD, Megraw M, Mockler TC. Alternative splicing in plants: directing traffic at the crossroads of adaptation and environmental stress. Curr Opin Plant Biol. 2015;24:125-35.

20. Engström PG, Steijger T, Sipos B, Grant GR, Kahles A, Rätsch G, Goldman N, Hubbard TJ, Harrow J, Guigó R, Bertone P, RGASP Consortium. Systematic evaluation of spliced alignment programs for RNA-seq data. Nat Methods. 2013;10:1185-91

21. Gan X, Stegle O, Behr J, Steffen JG, Drewe P, Hildebrand KL, Lyngsoe R, Schultheiss SJ, Osborne EJ, Sreedharan VT, et al. Multiple reference genomes and transcriptomes for Arabidopsis thaliana. Nature. 2011;477:419-23.

22. Guo G, Dondup D, Yuan X, Gu F, Wang D, Jia F, Lin Z, Baum M, Zhang J. Rare allele of HvLox-1 associated with lipoxygenase activity in barley (Hordeum vulgare L.). Theor Appl Genet. 2014;127:2095-103.

23. Guo W, Tzioutziou N, Stephen G, Milne I, Calixto C, Waugh R, Brown JWS, Zhang R. 3D RNA-seq - a powerful and flexible tool for rapid and accurate differential expression and alternative splicing analysis of RNA-seq data for biologists. bioRxiv. 2019; https://doi.org/10.1101/656686.

24. Haas BJ, Papanicolaou A, Yassour M, Grabherr M, Blood PD, Bowden J, Couger MB, Eccles D, Li B, Lieber M, MacManes MD, et al. De novo transcript sequence reconstruction from RNA-seq using the Trinity platform for reference generation and analysis. Nat Protoc. 2013:8:1494-512.

25. Hayer KE, Pizarro A, Lahens NF, Hogenesch JB, Grant GR. Benchmark analysis of algorithms for determining and quantifying full-length mRNA splice forms from RNA-seq data. Bioinformatics. 2015;31:3938-45.
26. International Barley Sequencing Consortium. A physical, genetic and functional sequence assembly of the barley genome. Nature. 2012;491:711-6.

27. Janiak A, Kwasniewski M, Sowa M, Gajek K, Żmuda K, Kościelniak J, Szarejko I. No time to waste: Transcriptome study reveals that drought tolerance in barley may be attributed to stressed-like expression patterns that exist before the occurrence of stress. Front Plant Sci. 2018;8:2212.

28. Kalyna M, Simpson CG, Syed NH, Lewandowska D, Marquez Y, Kusenda B, Marshall J, Fuller J, Cardle L, McNicol J, Dinh HQ, Barta A, Brown JWS. Alternative splicing and nonsense-mediated decay modulate expression of important regulatory genes in Arabidopsis. Nucleic Acids Res. 2012;40:2454-69.

29. Kim SH, Koroleva OA, Lewandowska D, Pendle AF, Clark GP, Simpson CG, Shaw PJ, Brown JWS. Aberrant mRNA transcripts and the nonsensemediated decay proteins UPF2 and UPF3 are enriched in the Arabidopsis nucleolus. Plant Cell. 2009;21:2045-57.

30. Kintlová M, Blavet N, Cegan R, Hobza R. Transcriptome of barley under three different heavy metal stress reaction. Genom Data. 2017;13:15-7.

31. Laloum T, Martin G, Duque P. Alternative splicing control of abiotic stress responses. Trends Plant Sci. 2018;23:140-50.

32. Lee Y, Rio DC. Mechanisms and regulation of alternative pre-mRNA splicing. Annu Rev Biochem. 2015;84:291-323.

33. Liu H, Liu H, Zhou L, Zhang Z, Zhang X, Wang M, Li H, Lin Z. Parallel domestication of the heading date 1 gene in cereals. Mol Biol Evol. 2015;32:2726-37.

34. Marquez Y, Brown JWS, Simpson C, Barta A, Kalyna M. Transcriptome survey reveals increased complexity of the alternative splicing landscape in Arabidopsis. Genome Res. 2012;22:1184-95.

35. Mascher M, Gundlach H, Himmelbach A, Beier S, Twardziok SO, Wicker T, Radchuk V, Dockter C, Hedley PE, Russell J, et al. A chromosome conformation capture ordered sequence of the barley genome. Nature. 2017:544:427-33.

36. Mastrangelo AM, Marone D, Laidò G, De Leonardis AM, De Vita P. Alternative splicing: enhancing ability to cope with stress via transcriptome plasticity. Plant Sci. 2012;185-186:40-9.

37. Matsumoto T, Tanaka T, Sakai H, Amano N, Kanamori H, Kurita K, Kikuta A, Kamiya K, Yamamoto M, lkawa $\mathrm{H}$, et al. Comprehensive sequence analysis of 24,783 barley full-length cDNAs derived from 12 clone libraries. Plant Physiol. 2011;156:20-8.

38. Mrízová K, Holasková E, Öz MT, Jiskrová E, Frébort I, Galuszka P. Transgenic barley: a prospective tool for biotechnology and agriculture. Biotechnol Adv. 2014;32:137-57.

39. Nilsen TW, Graveley BR. Expansion of the eukaryotic proteome by alternative splicing. Nature. 2010;463:457-63.

40. Ouyang S, Zhu W, Hamilton J, Lin H, Campbell M, Childs K, Thibaud-Nissen F, Malek RL, Lee Y, Zheng L, et al. 2007. The TIGR Rice Genome Annotation Resource: improvements and new features. Nucleic Acids Res. 35 (Database issue):D883-7.

41. Panahi B, Mohammadi SA, Ebrahimi Khaksefidi R, Fallah Mehrabadi J, Ebrahimie E. Genome-wide analysis of alternative splicing events in Hordeum vulgare: highlighting retention of intron-based splicing and its possible function through network analysis. FEBS Lett. 2015;589:3564-75.

42. Patro R, Duggal G, Love MI, Irizarry RA, Kingsford C. Salmon provides fast and bias-aware quantification of transcript expression. Nat Methods. 2017;14:417-9.

43. Pertea M, Pertea GM, Antonescu CM, Chang TC, Mendell JT, Salzberg SL. StringTie enables improved reconstruction of a transcriptome from RNA-seq reads. Nat Biotechnol. 2015;33:290-5.

44. Pham AT, Maurer A, Pillen K, Brien C, Dowling K, Berger B, Eglinton JK, March TJ. Genome-wide association of barley plant growth under drought stress using a nested association mapping population. BMC Plant Biol. 2019;19:134.

45. Reddy AS, Marquez Y, Kalyna M, Barta A. Complexity of the alternative splicing landscape in plants. Plant Cell. 2013;25:3657-83.

46. Ren P, Meng Y, Li B, Ma X, Si E, Lai Y, Wang J, Yao L, Yang K, Shang X, Wang H. Molecular Mechanisms of Acclimatization to Phosphorus Starvation and Recovery Underlying Full-Length Transcriptome Profiling in Barley (Hordeum vulgare L.). Front Plant Sci. 2018:9:500.

47. Russell J, Mascher M, Dawson IK, Kyriakidis S, Calixto C, Freund F, Bayer M, Milne I, Marshall-Griffiths T, Heinen S, et al. Exome sequencing of geographically diverse barley landraces and wild relatives gives insights into environmental adaptation. Nat Genet. 2016;48:1024-30.

48. Schindler S, Szafranski K, Hiller M, Ali GS, Palusa SG, Backofen R, Platzer M, Reddy AS. Alternative splicing at NAGNAG acceptors in Arabidopsis thaliana SR and SR-related protein-coding genes. BMC Genomics. 2008;9:159. 
49. Shi Y, Sha G, Sun X. Genome-wide study of NAGNAG alternative splicing in Arabidopsis. Planta. 2014;239:127-38.

50. Shirasu K, Lahaye T, Tan MW, Zhou F, Azevedo C, Schulze-Lefert P. A novel class of eukaryotic zinc-binding proteins is required for disease resistance signaling in barley and development in C. elegans. Cell. 1999; 99:355-66.

51. Simpson CG, Fuller J, Maronova M, Kalyna M, Davidson D, McNicol J, Barta A, Brown JWS. Monitoring changes in alternative precursor messenger RNA splicing in multiple gene transcripts. Plant J. 2008;53:1035-48.

52. Sokal RR, Rohlf FJ. The principles and practice of statistics in biological research. 3rd ed. New York: W. H. Freeman; 1995.

53. Soneson C, Love MI, Patro R, Hussain S, Malhotra D, Robinson MD. A junction coverage compatibility score to quantify the reliability of transcript abundance estimates and annotation catalogs. Life Sci Alliance. 2019;2: e201800175.

54. Staiger D, Brown JWS. Alternative splicing at the intersection of biological timing, development, and stress responses. Plant Cell. 2013;25:3640-56.

55. Szakonyi D, Duque P. Alternative splicing as a regulator of early plant development. Front Plant Sci. 2018;9:1174.

56. Thatcher SR, Zhou W, Leonard A, Wang BB, Beatty M, Zastrow-Hayes G, Zhao X, Baumgarten A, Li B. Genome-wide analysis of alternative splicing in Zea mays: landscape and genetic regulation. Plant Cell. 2014;26:3472-87.

57. Veeneman BA, Shukla S, Dhanasekaran SM, Chinnaiyan AM, Nesvizhskii Al. Two-pass alignment improves novel splice junction quantification. Bioinformatics. 2016;32:43-9.

58. Wu TD, Watanabe CK. GMAP: a genomic mapping and alignment program for mRNA and EST sequences. Bioinformatics. 2005;21:1859-75.

59. Zhang Q, Zhang X, Wang S, Tan C, Zhou G, Li C. Involvement of alternative splicing in barley seed germination. PLoS One. 2016a;11:e0152824.

60. Zhang Q, Zhang X, Pettolino F, Zhou G, Li C. Changes in cell wall polysaccharide composition, gene transcription and alternative splicing in germinating barley embryos. J Plant Physiol. 2016b;191:127-39.

61. Zhang R, Calixto CPG, Marquez Y, Venhuizen P, Tzioutziou NA, Guo W, Spensley M, Entizne JC, Lewandowska D, Ten Have S, et al. A high quality Arabidopsis transcriptome for accurate transcript-level analysis of alternative splicing. Nucleic Acids Res. 2017a;45:5061-73.

62. Zhang R, Calixto CP, Tzioutziou NA, James AB, Simpson CG, Guo W, Marquez Y, Kalyna M, Patro R, Eyras E, et al. AtRTD - a comprehensive reference transcript dataset resource for accurate quantification of transcript-specific expression in Arabidopsis thaliana. New Phytol. 2015; 208:96-101.

63. Zhang XN, Shi Y, Powers JJ, Gowda NB, Zhang C, Ibrahim HMM, Ball HB, Chen SL, Lu H, Mount SM. Transcriptome analyses reveal SR45 to be a neutral splicing regulator and a suppressor of innate immunity in Arabidopsis thaliana. BMC Genomics. 2017b;8:772.

\section{Publisher's Note}

Springer Nature remains neutral with regard to jurisdictional claims in published maps and institutional affiliations.

Ready to submit your research? Choose BMC and benefit from:

- fast, convenient online submission

- thorough peer review by experienced researchers in your field

- rapid publication on acceptance

- support for research data, including large and complex data types

- gold Open Access which fosters wider collaboration and increased citations

- maximum visibility for your research: over $100 \mathrm{M}$ website views per year

At $\mathrm{BMC}$, research is always in progress.

Learn more biomedcentral.com/submissions 